\title{
Architecting the implementation of WPB assessments at Indus Medical College, Pakistan
}

\author{
Memon, I.U. ${ }^{1}$, Memon, D.M.I. ${ }^{1}$, Khan, T.M. ${ }^{2}$ \\ ${ }^{1}$ Indus medical College, Pakistan, ${ }^{2}$ Sindh, Pakistan
}

\section{Background:}

The Indus Medical College (IMC), situated in a rural area of Pakistan, is a relatively newer one. We set and achieved many targets to make it at par with national and international standards. Since last few years trends in medical teaching and learning are shifting from traditional one to more innovative, learner-oriented methods. The tools aimed to assess trainees' clinical competence, behaviour and attitude conform with highest levels of Miller's pyramid. A group of such tools which had earned wide recognition is Work-Place Based Assessment (WPBA) that comprise various instruments including Direct Observation of Procedural Skills (DOPS), Mini Clinical Evaluation Exercise (mini-CEX) and Casebased Discussion $(\mathrm{CbD})$, which are used in real work place of learning. We plan to introduce and implement WPBA in our institution, with a strategy to ascertain the acceptability and preparedness of faculty and administration. To implement this a quantitative study was conducted amongst the faculty members of IMC.

\section{Methods:}

A questionnaire consisting of 8 items (with response option in affirmation or negation) was prepared and manually delivered to 148 respondents. The question were related to their knowledge of WPBS assessment, their prior experience, willingness to implement various tools of WPBA, practicability, probable acceptability by the trainees, willingness of administration to implement the methods, provide funds for the training of faculty, positive impact on assessment.

\section{Result:}

Out of 148 members, $126(85 \%)$ responded. A. $103(82 \%)$ respondents had prior knowledge of WPBA. B. $95(76 \%)$ had prior experience to assess trainees with various tools of WPBA. C. $100(80 \%)$ of responding faculty members were willing to implement. D. $92(73 \%)$ considered WPBA to be practical in the present circumstances of IMC. E. $87(68 \%)$ of the trainees were thought to accept the newer assessment methods. F. $102(81 \%)$ faculty members were hopeful about the willingness of administration. G. 97 (77\%) thought administration would provide funds for faculty training. H. $103(82 \%)$ presumed the implementation of WPBA would have positive impact.

\section{Conclusion:}

Largest number of present faculty members were aware of the WPBA tools. Though student's acceptability is anticipated at lower range, arrangement of workshops and seminars would make them aware of the true reflection of their knowledge, skills, and professionalism during training. Though most of the members presume positive willingness of the administration to introduce the methods but were sceptical about the provision of funds. In view of the positive response, we are highly confident that faculty would consider it as a positive step to improve the standards and thus make the institution compete with national and international medical institutions.

Keywords: Workplace Based Assessment 